\title{
On isolated, respectively consecutive large values of arithmetic functions
}

\author{
by
}

\author{
P. ERdős and A. SÁrközy (Budapest)
}

1. Throughout this paper we shall use the following notations: The set of positive integers is denoted by $\mathbb{N}$. If $f(x)=O(g(x))$, then we write $f(x) \ll g(x) . p_{i}$ denotes the $i$ th prime. $\omega(n)$ denotes the number of distinct prime divisors of $n$, while $\Omega(n)$ denotes the number of prime divisors of $n$ counted with multiplicity. $d(n)$ and $\sigma(n)$ denote the number, resp. sum of positive divisors of $n . \mu(n)$ is the Möbius function and $\varphi(n)$ denotes Euler's function. The smallest and greatest prime factors of $n$ are denoted by $p(n)$ and $P(n)$, respectively. If $f(n)$ is an arithmetic function and $x \geq 1$, then we write

$$
M(f, x)=\max _{n \leq x} f(n), \quad T(f, x)=\max _{n \leq x}(f(n-1)+f(n))
$$

and $G(f, x)$ will denote the greatest integer $G$ such that there is a number $n \in \mathbb{N}$ with $n \leq x$ and

$$
f(n)>\sum_{0<|i| \leq G} f(n+i) .
$$

In the first half of this paper (Sections 2-7) we will study isolated large values of the arithmetic functions $\omega(n), \Omega(n), d(n)$ and $\sigma(n)$, i.e., the function $G(f, x)$ with these four functions in place of $f$; see [1] and [5] for related results. (Since the first author studied a problem closely related to the estimate of $M(\varphi, x)$ in [4], we do not discuss the case $f(n)=\varphi(n)$ here.) In the second half of the paper we will study the converse of this problem by studying consecutive large values of these four functions, i.e., the function $T(f, x)$ with $\omega, \Omega, d$ and $\sigma$ in place of $f$. Note that $T(\Omega, x)$ and $T(\sigma, x)$ were studied by Erdös and Nicolas in [6]; here we will extend and sharpen their results.

Research partially supported by Hungarian National Foundation for Scientific Research, Grant No. 1901. 
2. First we will prove

THEOREM 1. There exist effectively computable positive constants $c_{1}$ and $c_{2}$ such that

$$
c_{1} \frac{\log x}{(\log \log x)^{2}}<G(\omega, x)<c_{2} \frac{\log x}{\log \log x \log \log \log x} .
$$

Note that almost certainly the lower bound gives the right order of magnitude of $G(\omega, x)$ but, unfortunately, we have not been able to show this; this is an interesting but, possibly, deep problem.

Proof. First we will prove the lower bound in (2.1). Define the positive integer $m=m(x)$ by

$$
\prod_{i=1}^{m-1} p_{i}<x^{1 / 2} \leq \prod_{i=1}^{m} p_{i}
$$

and write $P=\prod_{i=1}^{m} p_{i}$. Then by the prime number theorem we have

$$
m=\left(\frac{1}{2}+o(1)\right) \frac{\log x}{\log \log x}
$$

and

$$
x^{1 / 2} \leq P=p_{m} \prod_{i=1}^{m-1} p_{i} \ll x^{1 / 2} \log x .
$$

To prove the lower bound in (2.1), it suffices to show that writing

$$
G=\left[c_{1} \frac{\log x}{(\log \log x)^{2}}\right]+1,
$$

for $c_{1}$ small enough there is a $j \in \mathbb{N}$ such that

$$
j \leq x / P \quad \text { and } \quad \omega(j P)>\sum_{0<|i| \leq G} \omega(j P+i) .
$$

By (2.2) we have

$$
\begin{aligned}
\omega(j P) & \geq \omega(P)=m \\
& =\left(\frac{1}{2}+o(1)\right) \frac{\log x}{\log \log x} \quad \text { for all } j \in \mathbb{N} .
\end{aligned}
$$

On the other hand, if $\mathcal{P}_{1}, \mathcal{P}_{2}, \mathcal{P}_{3}$ denote the sets of primes $p$ with $p \leq G$, $G<p \leq x^{1 / 3}$, resp. $x^{1 / 3}<p \leq 2 x$, then by $(2.2),(2.3)$ and

$$
\sum_{p \leq y} \frac{1}{p}=\log \log y+C+O\left(\frac{1}{\log y}\right)
$$


we have

$$
\begin{aligned}
& \sum_{j \leq x / P} \sum_{0<|i| \leq G} \omega(j P+i) \\
& =\sum_{j \leq x / P} \sum_{0<|i| \leq G} \sum_{p \mid j P+i} 1 \\
& =\sum_{j \leq x / P} \sum_{p \in \mathcal{P}_{1}} \sum_{\substack{0<|i| \leq G \\
p \mid j P+i}} 1+\sum_{0<|i| \leq G} \sum_{p \in \mathcal{P}_{2}} \sum_{\substack{j \leq x / P \\
p \mid j P+i}} 1 \\
& +\sum_{j \leq x / P} \sum_{0<|i| \leq G} \sum_{\substack{p \in \mathcal{P}_{3} \\
p \mid j P+i}} 1 \\
& \ll \sum_{j \leq x / P} \sum_{p \leq G} \frac{G}{p}+\sum_{i=1}^{G} \sum_{G<p \leq x^{1 / 3}} \frac{x}{p P}+\sum_{j \leq x / P} \sum_{i=1}^{G} 1 \\
& \ll G \frac{x}{P}\left(\sum_{p \leq x^{1 / 3}} \frac{1}{p}+1\right) \ll G \frac{x}{P} \log \log x
\end{aligned}
$$

whence

$$
\min _{j \leq x / P} \sum_{0<|i| \leq G} \omega(j P+i) \ll G \log \log x<\frac{1}{3} \frac{\log x}{\log \log x}
$$

if $c_{1}$ is small enough. If the minimum in (2.7) is assumed for, say, $j=j_{0}$, then it follows from (2.5) and (2.7) that (2.4) holds with $j_{0}$ in place of $j$ and this completes the proof of the lower bound in (2.1).

To prove the upper bound in (2.1), it suffices to show that writing

$$
H=\left[c_{2} \frac{\log x}{\log \log x \log \log \log x}\right],
$$

for $c_{2}$ large enough we have

$$
\omega(n) \leq \sum_{0<|i| \leq H} \omega(n+i)
$$

for all $n \leq x$. Indeed, for $n \leq x$ we have

$$
\omega(n) \leq M(\omega, x)=(1+o(1)) \frac{\log x}{\log \log x}
$$

and, on the other hand, by (2.6) we have

$$
\sum_{0<|i| \leq H} \omega(n+i) \geq \sum_{p \leq H} \sum_{\substack{0<|i| \leq H \\ p \mid n+i}} 1 \gg H \log \log H .
$$


If $c_{2}$ is large enough, then (2.8) follows from (2.9) and (2.10), and this completes the proof of Theorem 1 .

3. $G(\Omega, x)$ can be estimated similarly:

THEOREM 2. There exist effectively computable positive constants $c_{3}, c_{4}$ such that

$$
c_{3} \frac{\log x}{\log \log x}<G(\Omega, x)<c_{4} \frac{\log x}{\log \log \log x} .
$$

Again, we think that the lower bound gives the right order of magnitude.

Proof. The proof is similar to the proof of Theorem 1, thus we will only sketch it. Write

$$
G=\left[c_{3} \frac{\log x}{\log \log x}\right]+1
$$

and define $u=u(x) \in \mathbb{N}$ by

$$
2^{u} \leq x^{1 / 2}<2^{u+1}
$$

so that $u=\left[\frac{\log x}{2 \log 2}\right]$. Then for $j \in \mathbb{N}, 1 \leq j \leq x / 2^{u}$ we have

$$
\Omega\left(j \cdot 2^{u}\right) \geq \Omega\left(2^{u}\right)=u \gg \log x
$$

and, on the other hand, it can be shown by an argument similar to the one in the proof of Theorem 1 that

$$
\sum_{j \leq x / 2^{u}} \sum_{0<|i| \leq G} \Omega\left(j \cdot 2^{u}+i\right) \ll \frac{x}{2^{u}} G \log \log x
$$

so that

$$
\min _{j \leq x / 2^{u}} \sum_{0<|i| \leq G} \Omega\left(j \cdot 2^{u}+i\right) \ll G \log \log x .
$$

If $c_{3}$ is small enough, then it follows from (3.2) and (3.3) that

$$
\min _{j \leq x / 2^{u}} \Omega\left(j \cdot 2^{u}\right)>\min _{j \leq x / 2^{u}} \sum_{0<|i| \leq G} \Omega\left(j \cdot 2^{u}+i\right),
$$

which proves the lower bound in (3.1).

To prove the upper bound in (3.1), observe that for $n \leq x$ we have

$$
\Omega(n) \leq M(\Omega, x)=\left[\frac{\log x}{\log 2}\right]
$$

and, writing

$$
H=\left[c_{4} \frac{\log x}{\log \log \log x}\right],
$$


we have

$$
\sum_{0<|i| \leq H} \Omega(n+i) \gg H \log \log H,
$$

which can be proved in the same way as (2.10). If $c_{4}$ is large enough, then it follows from (3.4) and (3.5) that

$$
\Omega(n) \leq \sum_{0<|i| \leq H} \Omega(n+i)
$$

(for all $n \leq x$ ), which implies the upper bound in (3.1).

4. The function $d(n)$ gives the most interesting and most difficult problem. In this case, we will prove

THEOREM 3. There are effectively computable absolute constants $x_{0}$ and $c_{5}$ such that for $x>x_{0}$ we have

$$
M(d, x) \exp \left(-11 \frac{\log x}{(\log \log x)^{3 / 2}}\right)<G(d, x)<c_{5} M(d, x) \frac{\log \log x}{\log x} .
$$

Note that it follows from the results of Ramanujan [9] that

$$
M(d, x)=\exp \left(\log 2 \frac{\log x}{\log \log x}+O\left(\frac{\log x}{(\log \log x)^{2}}\right)\right) .
$$

It follows from (4.1) and (4.2) that

$$
G(d, x)=\exp \left(\log 2 \frac{\log x}{\log \log x}+O\left(\frac{\log x}{(\log \log x)^{3 / 2}}\right)\right) .
$$

We expect the upper bound to be closer to the truth and perhaps we have

$$
G(d, x)>M(d, x)(\log x)^{-c} .
$$

5. The proof of the lower bound in (4.1) will be based on the following lemma:

LEMma 1. There is an effectively computable number $x_{0}$ such that if $x>x_{0}, a \in \mathbb{N}, Q \in \mathbb{N}$,

$$
\begin{gathered}
a Q \leq x, \\
Q>\exp \left(8 \frac{\log x}{(\log \log x)^{1 / 2}}\right),
\end{gathered}
$$

$b \in \mathbb{Z}$ and

$$
|b| \leq a,
$$

then there is a set $\mathcal{S}(a, b, Q)$ such that

$$
\mathcal{S}(a, b, Q) \subset\{1,2, \ldots, Q\},
$$




$$
|\mathcal{S}(a, b, Q)|<Q \exp \left(-2 \frac{\log x}{\log \log x}\right)
$$

and

$$
\sum_{\substack{i \leq Q \\ i \notin \mathcal{S}(a, b, Q)}} d(a i+b)<d(b) Q \exp \left(4 \frac{\log x}{(\log \log x)^{3 / 2}}\right) .
$$

Proof of Lemma 1. Clearly, for all $m, n \in \mathbb{N}$ we have

$$
d(m n) \leq d(m) d(n) .
$$

Thus writing $a=(a, b) a_{1}, b=(a, b) b_{1}$, for all $i \in \mathbb{N}$ we have

$$
d(a i+b) \leq d((a, b)) d\left(a_{1} i+b_{1}\right) \leq d(b) d\left(a_{1} i+b_{1}\right) .
$$

Set

$$
K=\frac{\log x}{(\log \log x)^{2}}, \quad L=\exp \left((\log \log x)^{3 / 2}\right),
$$

and for $n \in \mathbb{N}$ define $u=u(n), v=v(n), w=w(n)$ by

$$
n=u v w, \quad P(u) \leq K<p(v) \leq P(v) \leq L<p(w) .
$$

(If there is no prime $p$ with $p \mid n$ and $p \leq K, K<p \leq L$ or $L<p$, then we put $u=1, v=1$ and $w=1$, respectively.) Then for large $x$ and $n \leq 2 x$, by the prime number theorem clearly we have

$$
\begin{aligned}
d(u) & =\prod_{p \leq K, p^{\alpha} \| n} d\left(p^{\alpha}\right)=\prod_{p \leq K, p^{\alpha} \| n}(\alpha+1) \\
& \leq \prod_{p \leq K}\left(\frac{\log 2 x}{\log 2}+1\right)=\exp ((1+o(1)) \pi(K) \log \log x) \\
& =\exp \left((1+o(1)) \frac{\log x}{(\log \log x)^{2}}\right)<\exp \left(2 \frac{\log x}{(\log \log x)^{2}}\right) .
\end{aligned}
$$

Moreover, it follows from the definition of $w$ that for $n \leq 2 x$ we have

$$
2 x \geq n \geq w=\prod_{p>L, p^{\alpha} \| n} p^{\alpha} \geq \prod_{p>L, p^{\alpha} \| n} L^{\alpha}=L^{\Omega(w)},
$$

whence

$$
\Omega(w) \leq \frac{\log 2 x}{\log L}<2 \frac{\log x}{(\log \log x)^{3 / 2}} .
$$

For all $m \in \mathbb{N}$ we have $d(m) \leq 2^{\Omega(m)}$. Thus from (5.11) we obtain

$$
d(w) \leq \exp \left(2 \log 2 \frac{\log x}{(\log \log x)^{3 / 2}}\right) .
$$


By (5.1), (5.3) and (5.7), it follows from (5.8), (5.9), (5.10) and (5.12) that for all $i \leq Q$ we have

$$
\begin{aligned}
d(a i+b) & \leq d(b) d\left(a_{1} i+b_{1}\right) \\
& \leq d(b) d\left(u\left(a_{1} i+b_{1}\right)\right) d\left(v\left(a_{1} i+b_{1}\right)\right) d\left(w\left(a_{1} i+b_{1}\right)\right) \\
& <d(b) d\left(v\left(a_{1} i+b_{1}\right)\right) \exp \left(3 \frac{\log x}{(\log \log x)^{3 / 2}}\right) \quad(\text { for } i \leq Q)
\end{aligned}
$$

Now write

$$
z=\left[7 \frac{\log x}{(\log \log x)^{2}}\right]
$$

and let $\mathcal{S}(a, b, Q)$ denote the set of the positive integers $i$ such that $i \leq Q$ and

$$
\Omega\left(v\left(a_{1} i+b_{1}\right)\right)>z .
$$

Then (5.4) holds trivially. Write $v\left(a_{1} i+b_{1}\right)$ as the product of a square and a square-free number:

$$
v\left(a_{1} i+b_{1}\right)=r^{2} q \quad(r, q \in \mathbb{N},|\mu(q)|=1) .
$$

It follows from (5.14) and (5.15) that either

$$
\Omega(r) \geq z / 3
$$

or

$$
\Omega(q)=\omega(q) \geq z / 3 .
$$

Let $\mathcal{T}_{1}$ denote the set of the integers $t$ with $t \in \mathbb{N}, K<p(t) \leq P(t) \leq L$, $\Omega(t)=[z / 3]$, and let $\mathcal{T}_{2}$ denote the set of square-free elements of $\mathcal{T}_{1}$ so that for $t \in \mathcal{T}_{2}$ we have $K<p(t) \leq P(t) \leq L, \Omega(t)=\omega(t)=[z / 3]$. Then for $i \in \mathcal{S}(n, b, Q)$ either there is an integer $t$ such that $t \in \mathcal{T}_{1}$ and $t^{2} \mid a_{1} i+b_{1}$, or there is an integer $t$ such that $t \in \mathcal{T}_{2}$ and $t \mid a_{2} i+b_{2}$ (in fact, if (5.16) holds, then $t$ can be chosen as any divisor of $r$ with $\Omega(t)=[z / 3]$, while if (5.17) holds, then $t$ can be any divisor of $q$ with $\Omega(t)=\omega(t)=[z / 3])$. It follows that

$$
\begin{aligned}
|\mathcal{S}(a, b, Q)| \leq & \sum_{t \in \mathcal{T}_{1}}\left|\left\{i: i \leq Q, t^{2} \mid a_{1} i+b_{1}\right\}\right| \\
& +\sum_{t \in \mathcal{T}_{2}}\left|\left\{i: i \leq Q, t \mid a_{1} i+b_{1}\right\}\right| .
\end{aligned}
$$

Clearly, if $t \in \mathcal{T}_{1} \cup \mathcal{T}_{2}$, then we have

$$
\begin{aligned}
t & =\prod_{p^{\alpha} \| t} p^{\alpha}>K^{\Omega(t)}=K^{[z / 3]}=\exp \left((1+o(1)) \frac{z}{3} \log K\right) \\
& =\exp \left((1+o(1)) \frac{7}{3} \frac{\log x}{\log \log x}\right) \quad\left(\text { for } t \in \mathcal{T}_{1} \cup \mathcal{T}_{2}\right)
\end{aligned}
$$


and

$$
\begin{aligned}
t & =\prod_{p^{\alpha} \| t} p^{\alpha} \leq L^{\Omega(t)}=L^{[z / 3]}=\exp \left((1+o(1)) \frac{z}{3} \log L\right) \\
& =\exp \left((1+o(1)) \frac{7}{3} \frac{\log x}{(\log \log x)^{1 / 2}}\right) \\
& <\left(3 \frac{\log x}{(\log \log x)^{1 / 2}}\right)<Q^{1 / 2} \quad\left(\text { for } t \in \mathcal{T}_{1} \cup \mathcal{T}_{2}\right) .
\end{aligned}
$$

By $\left(a_{1}, b_{1}\right)=1$, for all $d \in\{1,2, \ldots, Q\}$ we have

$$
\left|\left\{i: i \leq Q, d \mid a_{1} i+b_{1}\right\}\right| \leq \frac{Q}{d}+1 \leq 2 \frac{Q}{d} \quad(\text { for } d \leq Q) .
$$

It follows from (5.18), (5.20) and (5.21) that

$$
|\mathcal{S}(a, b, Q)| \leq \sum_{t \in \mathcal{T}_{1}} 2 \frac{Q}{t^{2}}+\sum_{t \in \mathcal{T}_{2}} 2 \frac{Q}{t}=2 Q\left(\sum_{t \in \mathcal{T}_{1}} \frac{1}{t^{2}}+\sum_{t \in \mathcal{T}_{2}} \frac{1}{t}\right) .
$$

By (5.19) we have

$$
\sum_{t \in \mathcal{T}_{1}} \frac{1}{t^{2}} \leq \sum_{t>\exp \left((1+o(1)) \frac{7}{3} \frac{\log x}{\log \log x}\right)} \frac{1}{t^{2}}=\exp \left(-(1+o(1)) \frac{7}{3} \frac{\log x}{\log \log x}\right)
$$

Moreover, by (2.6) and Stirling's formula we have

$$
\begin{aligned}
\sum_{t \in \mathcal{T}_{2}} \frac{1}{t} & =\sum_{K<p_{i_{1}}<p_{i_{2}}<\ldots<p_{i_{[z / 3]}} \leq L} \frac{1}{p_{i_{1}} p_{i_{2}} \ldots p_{i_{[z / 3]}}} \\
& <\frac{1}{([z / 3]) !}\left(\sum_{p \leq L} \frac{1}{p}\right)^{[z / 3]}=\exp \left(-(1+o(1)) \frac{7}{3} \frac{\log x}{\log \log x}\right) .
\end{aligned}
$$

(5.5) follows from (5.22), (5.23) and (5.24).

It remains to show that (5.6) also holds. By (5.13) we have

$$
\begin{aligned}
& \sum_{i \leq Q, i \notin \mathcal{S}(a, b, Q)} d(a i+b) \\
& \quad<d(b) \exp \left(3 \frac{\log x}{(\log \log x)^{3 / 2}}\right) \sum_{i \leq Q, i \notin \mathcal{S}(a, b, Q)} d\left(v\left(a_{1} i+b_{1}\right)\right) .
\end{aligned}
$$

Here the last sum is

$$
\sum_{i \leq Q, i \notin \mathcal{S}(a, b, Q)}\left|\left\{d: d \mid v\left(a_{1} i+b_{1}\right)\right\}\right| .
$$

If $d \mid v\left(a_{1} i+b_{1}\right)$ for some $i \leq Q, i \notin \mathcal{S}(a, b, Q)$, then, by the definitions of $v(n)$ and $\mathcal{S}(a, b, Q)$, for all $p \mid d$ we have $p \leq L$; moreover, $\Omega(d) \leq z$. Thus 
by (5.2), for such a $d$ we have

$$
d=\prod_{p^{\alpha} \| d} p^{\alpha} \leq L^{\Omega(d)} \leq L^{z}=\exp \left((1+o(1)) 7 \frac{\log x}{(\log \log x)^{1 / 2}}\right)<Q
$$

so that for this fixed $d$, by (5.21) we have

$$
\left|\left\{i: i \leq Q, i \notin \mathcal{S}(a, b, Q), d \mid a_{1} i+b_{1}\right\}\right| \leq 2 \frac{Q}{d},
$$

whence, by Mertens' formula,

$$
\begin{aligned}
\sum_{i \leq Q, i \notin \mathcal{S}(a, b, Q)} d\left(v \left(a_{1} i+\right.\right. & \left.\left.b_{1}\right)\right) \\
& \leq \sum_{P(d) \leq L, \Omega(d) \leq z} 2 \frac{Q}{d}<2 Q \sum_{P(d) \leq L} \frac{1}{d} \\
& =2 Q \prod_{p \leq L} \sum_{\alpha=0}^{\infty} \frac{1}{p^{\alpha}}=2 Q \prod_{p \leq L}\left(1-\frac{1}{p}\right)^{-1} \\
& \ll Q \log L=Q(\log \log x)^{3 / 2} .
\end{aligned}
$$

(5.6) follows from (5.25) and (5.26), and this completes the proof of the lemma.

6. Completion of the proof of Theorem 3. First we will prove the lower bound in (4.1). Set

$$
Q=\left[\exp \left(8 \frac{\log x}{(\log \log x)^{1 / 2}}\right)\right]+1
$$

and

$$
G=\left[M(d, x) \exp \left(-11 \frac{\log x}{(\log \log x)^{3 / 2}}\right)\right]+1 .
$$

Define the positive integer $m$ by

$$
\prod_{i=1}^{m} p_{i} \leq \frac{x}{Q}<\prod_{i=1}^{m+1} p_{i}
$$

so that, by the prime number theorem, we have

$$
\begin{aligned}
m & =\frac{\log (x / Q)}{\log \log (x / Q)}\left(1+O\left((\log \log (x / Q))^{-1}\right)\right) \\
& =\frac{\log x}{\log \log x}\left(1-8(\log \log x)^{-1 / 2}+O\left((\log \log x)^{-1}\right)\right) .
\end{aligned}
$$


Write

$$
P=\prod_{i=1}^{m} p_{i} .
$$

In order to prove the lower bound in (4.1), it suffices to show that for large $x$ there is a $j \in \mathbb{N}$ such that

$$
j \leq x / P \quad \text { and } \quad d(j P)>\sum_{0<|i| \leq G} d(j P+i) .
$$

By (4.2) and (6.4), for large $x$ we have

$$
\begin{aligned}
& d(j P) \\
& \geq d(P)=2^{m} \\
& =\exp \left(\log 2 \frac{\log x}{\log \log x}\left(1-8(\log \log x)^{-1 / 2}+O\left((\log \log x)^{-1}\right)\right)\right) \\
& =M(d, x) \exp \left(-(1+o(1)) 8 \log 2 \frac{\log x}{(\log \log x)^{3 / 2}}\right) \\
& \quad \text { (for all } j \leq x / P) .
\end{aligned}
$$

On the other hand, let $\mathcal{S}=\bigcup_{0<|b| \leq G} \mathcal{S}(P, b, Q)$ where $G, P, Q$ are defined by (6.2), (6.5) and (6.1), respectively, and the set $\mathcal{S}(P, b, Q)$ is defined in Lemma 1 . Then by Lemma 1 and (4.2), for large $x$ we have

$$
|\mathcal{S}| \leq \sum_{0<|b| \leq Q}|\mathcal{S}(P, b, Q)| \leq 2 G Q \exp \left(-2 \frac{\log x}{\log \log x}\right)<\frac{Q}{2} .
$$

Let $\mathcal{J}=\{1,2, \ldots, Q\} \backslash \mathcal{S}$ so that by (6.8) we have

$$
|\mathcal{J}|=Q-|\mathcal{S}|>Q / 2 \text {. }
$$

Then by Lemma 1 and the definition of $\mathcal{J}$ we have

$$
\begin{aligned}
\sum_{i \in \mathcal{J}} \sum_{0<|b| \leq G} d(P i+b) & \leq \sum_{0<|b| \leq G} \sum_{i \leq Q, i \notin \mathcal{S}(P, b, Q)} d(P i+b) \\
& <\sum_{0<|b| \leq G} d(b) Q \exp \left(4 \frac{\log x}{(\log \log x)^{3 / 2}}\right) \\
& =Q \exp \left(4 \frac{\log x}{(\log \log x)^{3 / 2}}\right) \sum_{0<|b| \leq G} d(b) \\
& \ll Q \exp \left(4 \frac{\log x}{(\log \log x)^{3 / 2}}\right) G \log G
\end{aligned}
$$

It follows from (6.9) and (6.10) that there is a $j \in \mathbb{N}$ with

$$
j \leq Q \leq x / P
$$


such that

$$
\begin{aligned}
\sum_{0<|b| \leq G} d(P j+b) & \ll \exp \left(4 \frac{\log x}{(\log \log x)^{3 / 2}}\right) G \log G \\
& <M(d, x) \exp \left(-6 \frac{\log x}{(\log \log x)^{3 / 2}}\right) .
\end{aligned}
$$

(6.6) follows from (6.7), (6.11) and (6.12), and this completes the proof of the lower bound in (4.1).

To prove the upper bound in (4.1), write

$$
H=\left[c_{6} \frac{\log \log x}{\log x} M(d, x)\right] .
$$

Then for sufficiently large $c_{6}$ and all $n \leq x$, by (4.2) we have

$$
\begin{aligned}
\sum_{0<|i| \leq H} d(n+i) & =\sum_{0<|i| \leq H} \sum_{d \mid n+i} 1 \geq \sum_{0<|i| \leq H} \sum_{\substack{d \mid n+i \\
d \leq H}} 1 \\
& =\sum_{d=1}^{H} \sum_{\substack{0<|i| \leq H \\
d \mid n+i}} 1 \gg \sum_{d=1}^{H} \frac{H}{d} \\
& \gg H \log H>M(d, x) \geq d(n)
\end{aligned}
$$

which proves the upper bound in (4.1).

7. The sharpest estimate can be given for $G(\sigma, x)$ :

THEOREM 4. We have

$$
G(\sigma, x)=(1+o(1)) 3 \pi^{-2} e^{\gamma} \log \log x
$$

where $\gamma=0.57722 \ldots$ is Euler's constant.

Proof. First we will show that

$$
G(\sigma, x) \geq(1+o(1)) 3 \pi^{-2} e^{\gamma} \log \log x .
$$

Let $0<\varepsilon<1$, and write

$$
G=\left[(1-\varepsilon) 3 \pi^{-2} e^{\gamma} \log \log x\right] .
$$

Moreover, for $p \leq \frac{1}{2} \log x$ define $\alpha_{p}$ by

$$
p^{\alpha_{p}-1} \leq(\log x)^{1 / 2}<p^{\alpha_{p}}
$$

and let

$$
R=\prod_{p \leq \frac{1}{2} \log x} p^{\alpha_{p}}
$$


Then by the prime number theorem we have

$$
\begin{aligned}
R & =\prod_{p \leq(\log x)^{1 / 2}} p^{\alpha_{p}-1} \prod_{p \leq \frac{1}{2} \log x} p \leq \prod_{p \leq(\log x)^{1 / 2}}(\log x)^{1 / 2} \prod_{p \leq \frac{1}{2} \log x} p \\
& =\exp \left(\frac{1}{2} \log \log x \pi\left((\log x)^{1 / 2}\right)+\left(\frac{1}{2}+o(1)\right) \log x\right)=\exp \left(\left(\frac{1}{2}+o(1)\right) \log x\right),
\end{aligned}
$$

whence

$$
R \leq x .
$$

To prove (7.2), it suffices to show that for all $\varepsilon$ and $x>x_{0}(\varepsilon)$ there is a $j \in \mathbb{N}$ such that

$$
j \leq x / R \quad \text { and } \quad \sigma(j R)>\sum_{0<|i| \leq G} \sigma(j R+i) .
$$

For all $m, n \in \mathbb{N}$ we have

$$
\frac{\sigma(m n)}{m n} \geq \frac{\sigma(m)}{m} .
$$

Thus by Mertens' formula, for all $j \in \mathbb{N}$ we have

$$
\begin{aligned}
& \frac{\sigma(j R)}{j R} \\
\geq & \frac{\sigma(R)}{R}=\prod_{p \leq \frac{1}{2} \log x} \sigma\left(p^{\alpha_{p}}\right) p^{-\alpha_{p}} \\
= & \prod_{p \leq \frac{1}{2} \log x}\left(\sum_{\alpha=0}^{\alpha_{p}} p^{-k}\right)=\prod_{p \leq \frac{1}{2} \log x}\left(1-\frac{1}{p}\right)^{-1}\left(1-p^{-\left(\alpha_{p}+1\right)}\right) \\
> & \prod_{p \leq \frac{1}{2} \log x}\left(1-\frac{1}{p}\right)^{-1} \prod_{p \leq(\log x)^{1 / 4}}\left(1-(\log x)^{-1 / 2}\right) \prod_{(\log x)^{1 / 4}<p}\left(1-p^{-2}\right) \\
= & (1+o(1)) e^{\gamma} \log \log x\left(1-(\log x)^{-1 / 2}\right)^{\pi\left((\log x)^{1 / 4}\right)}(1+o(1)) \\
= & (1+o(1)) e^{\gamma} \log \log x .
\end{aligned}
$$

On the other hand, uniformly for $j \in \mathbb{N}, j \leq x / R$ we have

$$
\begin{aligned}
\frac{1}{j R} \sum_{0<|i| \leq G} \sigma(j R+i) & =\sum_{0<|i| \leq G} \frac{\sigma(j R+i)}{j R+i} \cdot \frac{j R+i}{j R} \\
& =\left(1+O\left(\frac{G}{R}\right)\right) \sum_{0<|i| \leq G} \frac{\sigma(j R+i)}{j R+i} \\
& =(1+o(1)) \sum_{0<|i| \leq G} \frac{\sigma(j R+i)}{j R+i} .
\end{aligned}
$$


Now consider

$$
\sum_{j \leq x / R} \sum_{0<|i| \leq G} \frac{\sigma(j R+i)}{j R+i}=\sum_{j \leq x / R} \sum_{0<|i| \leq G} \sum_{d \mid j R+i} \frac{1}{d} .
$$

We split this sum into two parts according to the magnitude of $d$. First we have

$$
\begin{aligned}
\sum_{d \leq G} \sum_{j \leq x / R} \sum_{\substack{0<|i| \leq G \\
d \mid j R+i}} \frac{1}{d} & =\sum_{d \leq G} \frac{1}{d} \sum_{j \leq x / R} \sum_{\substack{0<|i| \leq G \\
d \mid j R+i}} 1 \\
& \leq \sum_{d \leq G} \frac{1}{d} \sum_{j \leq x / R} 2\left(\frac{G}{d}+1\right) \\
& =2\left[\frac{x}{R}\right] \sum_{d \leq G}\left(\frac{G}{d^{2}}+\frac{1}{d}\right) \\
& <\left[\frac{x}{R}\right]\left(\frac{\pi^{2}}{3} G+O(\log G)\right) .
\end{aligned}
$$

Moreover, for $G<d \leq 2 x, 0<|i| \leq G$ we have

$$
\begin{aligned}
\sum_{\substack{j \leq x / R \\
d \mid j R+i}} \frac{1}{d} & =\frac{1}{d}\left|\left\{j: j \leq x / R, \frac{d}{(d,(R, i))} \mid\left(j \frac{R}{(R, i)}+\frac{i}{(R, i)}\right)\right\}\right| \\
& \leq \frac{1}{d}\left(\frac{x(d,(R, i))}{R d}+1\right) \leq \frac{1}{d}\left(\frac{x(d, i)}{R d}+1\right) .
\end{aligned}
$$

Thus writing $(d, i)=r, d=r s, i=r t$, we have

$$
\begin{aligned}
\sum_{G<d \leq 2 x} \sum_{0<|i| \leq G} \sum_{\substack{j \leq x / R \\
d \mid j R+i}} \frac{1}{d} & \leq \sum_{G<d \leq 2 x}\left(\frac{2 G}{d}+2 \frac{x}{R} \sum_{i=1}^{G} \frac{(d, i)}{d^{2}}\right) \\
& \ll G \log x+\frac{x}{R} \sum_{r=1}^{G} \sum_{t \leq G / r} \sum_{G / r<s \leq 2 x / r} \frac{r}{r^{2} s^{2}} \\
& \ll G \log x+\frac{x}{R} \sum_{r=1}^{G} \sum_{t \leq G / r} \frac{1}{G} \\
& \ll G \log x+\frac{x}{R} \log G \ll \frac{x}{R} \log G .
\end{aligned}
$$

By (7.9), (7.10) and (7.11), we have

$$
\sum_{j \leq x / R} \sum_{0<|i| \leq G} \frac{\sigma(j R+i)}{j R+i} \leq\left[\frac{x}{R}\right](1+o(1)) \frac{\pi^{2}}{3} G .
$$


It follows that there is a $j \leq x / R$ with

$$
\sum_{0<|i| \leq G} \frac{\sigma(j R+i)}{j R+i} \leq(1+o(1)) \frac{\pi^{2}}{3} G .
$$

Combining this with (7.7), we obtain

$$
\begin{aligned}
\frac{1}{j R} \sum_{0<|i| \leq G} \sigma(j R+i) & \leq(1+o(1)) \frac{\pi^{2}}{3} G \\
& =(1+o(1))(1-\varepsilon) e^{\gamma} \log \log x .
\end{aligned}
$$

(7.5) follows from (7.7) and (7.12), which completes the proof of (7.2).

To prove that

$$
G(\sigma, x) \leq(1+o(1)) 3 \pi^{-2} e^{\gamma} \log \log x,
$$

we have to show that if $\varepsilon>0$ and we write

$$
H=\left[(1+\varepsilon) 3 \pi^{-2} e^{\gamma} \log \log x\right],
$$

then for $x>x_{0}(\varepsilon), H<n \leq x$ we have

$$
\sigma(n)<\sum_{0<|i| \leq H} \sigma(n+i)
$$

or, in equivalent form,

$$
\frac{\sigma(n)}{n}<\frac{1}{n} \sum_{0<|i| \leq H} \sigma(n+i) .
$$

Since (7.13) is nearly trivial for $n / H=O(1)$, we may assume that $n / H \rightarrow$ $\infty$. It is well-known (and, by using Mertens' formula, it can be shown easily) that for $n \leq x$ we have

$$
\frac{\sigma(n)}{n} \leq(1+o(1)) e^{\gamma} \log \log x .
$$

On the other hand, for $n / H \rightarrow \infty$ we have

$$
\begin{aligned}
\frac{1}{n} \sum_{0<|i| \leq H} \sigma(n+i) & =(1+o(1)) \sum_{0<|i| \leq H} \frac{\sigma(n+i)}{n+i} \\
& =(1+o(1)) \sum_{0<|i| \leq H} \sum_{d \mid n+i} \frac{1}{d} \\
& \geq(1+o(1)) \sum_{d=1}^{H} \frac{1}{d} \sum_{\substack{0<|i| \leq H \\
d \mid n+i}} 1
\end{aligned}
$$




$$
\begin{aligned}
& =(1+o(1)) \sum_{d=1}^{H} \frac{1}{d}\left(2 \frac{H}{d}+O(1)\right) \\
& =(1+o(1))\left(2 H \sum_{d=1}^{H} \frac{1}{d^{2}}+O\left(\sum_{d=1}^{H} \frac{1}{d}\right)\right) \\
& =(1+o(1))\left(\frac{\pi^{2}}{3} H+O(\log H)\right) \\
& =(1+o(1))(1+\varepsilon) e^{\gamma} \log \log x .
\end{aligned}
$$

If $x>x_{0}(\varepsilon)$, then (7.13) follows from (7.14) and (7.15), which completes the proof of the theorem.

8. Our results and methods presented above are of different nature for each of the four functions $\omega(n), \Omega(n), d(n)$ and $\sigma(n)$. The following facts explain some of these differences:

If $f(n)$ is an arithmetic funcion and $n \in \mathbb{N}$, then let $g(f, n)$ denote the smallest integer $g$ such that

$$
f(n)<\sum_{i=1}^{g} f(n+i) .
$$

If $f(n)=\omega(n), \Omega(n), d(n)$ or $\sigma(n)$, then the density of the integers $n$ with $f(n)<f(n+1)$ is $1 / 2$. Moreover, if $f(n)=\omega(n)$ or $\Omega(n)$, then for almost all $n$ we have $f(n)=(1+o(1)) \log \log n$. It follows that for almost all $n$ we have $g(\omega, n) \leq 2$ and $g(\Omega, n) \leq 2$ and, indeed, the density of the integers $n$ with $g(\omega, n)=1, g(\omega, n)=2, g(\Omega, n)=1$, resp. $g(\Omega, n)=2$ is $1 / 2$.

This is not so for the functions $\sigma(n)$ and $d(n)$. Indeed, considering the function $d(n)$, for every $t \in \mathbb{N}$ the density of the integers $n$ with $g(d, n)=t$ is $1 / 2^{t}$, and for almost all $n$ we have $d(n+g(d, n))>d(n)$. If we consider the function $\sigma(n)$, then again, the density of the integers $n$ with $g(\sigma, n)=t$ is positive for all $n \in \mathbb{N}$ but, on the other hand, the density of the integers $n$ with $\sigma(n+g(\sigma, n))>\sigma(n)$ is $<1$ (and $>0$ ).

One might like to study the analogous questions for $f(n)=P(n)$. We can show that

$$
\lim _{x \rightarrow \infty} G(P, x)=\infty
$$

it would be interesting to estimate $g(P, x)$.

9. In the rest of this paper we will study consecutive large values of arithmetic functions. Erdős and Nicolas [6] proved that

$$
T(\Omega, x)=(1+o(1)) \frac{\log x}{\log 2}
$$


where the error term is ineffective and

$$
T(\sigma, x) \leq(1+o(1)) e^{\gamma} x \log \log x
$$

where $\gamma$ is Euler's constant. In this paper our goal is to extend and sharpen these results. Note that the estimate of $T(\omega, x)$ seems to be very difficult, in particular, we have not been able to show that

$$
\lim _{x \rightarrow \infty} \sup (T(\omega, x)-M(\omega, x))=\infty
$$

(which is certainly true), and the study of $T(d, x)$ seems to be even more difficult.

In Sections 9 and 10, we will study $T(\Omega, x)$, while in Sections 11 and 12 we will estimate $T(\sigma, x)$. First we give a lower bound for $T(\Omega, x)$ :

TheOREM 5. For all $\varepsilon>0$ there are infinitely many numbers $x \in \mathbb{N}$ such that

$$
\begin{aligned}
T(\Omega, x) & >M(\Omega, x)+\exp \left((\log 2-\varepsilon) \frac{\log \log x}{\log \log \log x}\right) \\
& =\left[\frac{\log x}{\log 2}\right]+\exp \left((\log 2-\varepsilon) \frac{\log \log x}{\log \log \log x}\right) .
\end{aligned}
$$

Proof. The proof will be based on the following result of A. S. Bang [2] (see also [3, p. 385]):

Lemma 2. If $n \in \mathbb{N}$ and $n \neq 1,6$, then there is a prime $p$ such that $p \mid 2^{n}-1$ but $p \nmid 2^{m}-1$ for $m=1,2, \ldots, n-1$.

To prove (9.3), consider a highly composite number $n$, i.e., assume that

$$
d(n)=M(d, n) \quad\left(=\exp \left((1+o(1)) \log 2 \frac{\log n}{\log \log n}\right)\right) .
$$

Write $x=2^{n}$. Then by Lemma 2 we have

$$
\begin{aligned}
T(\Omega, x) & \geq \Omega(x-1)+\Omega(x)=\Omega\left(2^{n}-1\right)+n \\
& \geq\left|\left\{i:\left(2^{i}-1\right) \mid\left(2^{n}-1\right), i \neq 1,6\right\}\right|+M(\Omega, x) \\
& \geq(d(n)-2)+M(\Omega, x) \\
& =\exp \left((1+o(1)) \log 2 \frac{\log n}{\log \log n}\right)+M(\Omega, x) \\
& >\exp \left((\log 2-\varepsilon) \frac{\log \log x}{\log \log \log x}\right)+M(\Omega, x)
\end{aligned}
$$

for $x$ large enough, which proves (9.3).

10. In this section we will give upper bounds for consecutive values of the $\Omega$ function.

Erdős and Nicolas proved (9.1) by using a result of Ridout. Next we will show by using a result of Mahler that (9.1) can be extended to $k$ consecutive values of the $\Omega$ function. 
TheOREM 6. For every $\varepsilon>0$ and $k \in \mathbb{N}$ there is an (ineffective) number $x_{0}=x_{0}(\varepsilon, k)$ such that for $x>x_{0}$ we have

$$
\begin{array}{r}
\left(\left[\frac{\log x}{\log 2}\right] \leq\right) \max _{n \leq x}(\Omega(n-k+1)+\Omega(n- \\
<+2)+\ldots+\Omega(n)) \\
<\left(\frac{1}{\log 2}+\varepsilon\right) \log x .
\end{array}
$$

Pr o of. If $\mathcal{S}=\left\{q_{1}, q_{2}, \ldots, q_{t}\right\}$ is a finite set of prime numbers and $a \in \mathbb{N}$, then denote the largest divisor of $a$ composed solely of powers of primes form $\mathcal{S}$ by $[a]_{\mathcal{S}}$ so that $a$ can be represented in the form

$$
a=[a]_{\mathcal{S}} v, \quad v \in \mathbb{N}, \quad\left(v, \prod_{p \in \mathcal{S}} p\right)=1 .
$$

The proof of (10.1) will be based on the following result of Mahler [8, p. 159, Theorem 5, II]:

Lemma 3. If $\mathcal{S}$ is a finite set of prime numbers, $k \in \mathbb{N}$ and $\varepsilon>0$, then there is an (ineffective) number $n_{0}=n_{0}(\mathcal{S}, k, \varepsilon)$ such that for $n>n_{0}$ we have

$$
[(n-k+1)(n-k+2) \ldots n]_{\mathcal{S}}<n^{1+\varepsilon} .
$$

In order to prove (10.1), clearly it suffices to show that for $n>n_{1}(k, \varepsilon)$ we have

$$
\Omega(n-k+1)+\Omega(n-k+2)+\ldots+\Omega(n)<\left(\frac{1}{\log 2}+\varepsilon\right) \log n .
$$

Define $t$ by

$$
\frac{k}{\log p_{t+1}} \leq \frac{\varepsilon}{2}<\frac{k}{\log p_{t}}
$$

and let $\mathcal{S}=\left\{p_{1}, p_{2}, \ldots, p_{t}\right\}$. Write $u=[(n-k+1)(n-k+2) \ldots n]_{\mathcal{S}}$ and define $v$ by

$$
(n-k+1)(n-k+2) \ldots n=u v
$$

so that $v \in \mathbb{N}$,

$$
\left(v, \prod_{i=1}^{t} p_{i}\right)=1
$$

and

$$
\Omega(n-k+1)+\Omega(n-k+2)+\ldots+\Omega(n)=\Omega(u)+\Omega(v) .
$$

By Lemma 3 (with $\varepsilon / 4$ in place of $\varepsilon$ ), for $n>n_{2}(k, \varepsilon)$ we have

$$
u<n^{1+\varepsilon / 4} \text {, }
$$


whence

$$
\Omega(u) \leq\left[\frac{\log n^{1+\varepsilon / 4}}{\log 2}\right]<\left(\frac{1}{\log 2}+\frac{\varepsilon}{2}\right) \log n .
$$

Moreover, by (10.5) and (10.6) we have

$$
n^{k} \geq u v \geq v=\prod_{p^{\alpha} \| v} p^{\alpha} \geq p_{t+1}^{\Omega(v)}
$$

whence

$$
\Omega(v) \leq \frac{k \log n}{\log p_{t+1}} .
$$

(10.3) follows from (10.4), (10.7), (10.8) and (10.9), and this completes the proof of Theorem 6 .

Since both (9.1) and Theorem 6 are ineffective, one might like to give an effective upper bound for consecutive values of the $\Omega$ function. Here we restrict ourselves to the case $k=2$ (the case $k>2$ could be handled similarly).

THEOREM 7. There are effectively computable positive numbers $n_{3}$ and $c_{7}$ such that for $n>n_{3}, n \in \mathbb{N}$ we have

$$
\Omega(n-1)+\Omega(n)<\left(\frac{1}{\log 2}+\frac{1}{\log 3}-c_{7}\right) \log n .
$$

Proof. The proof will be based on the following result of Stewart [10, Theorem 2]:

Lemma 4. If $\mathcal{S}=\left\{q_{1}, q_{2}, \ldots, q_{r}\right\}$ is a finite set of distinct prime numbers and $k \in \mathbb{N}$, then there are positive numbers $c_{8}$ and $c_{9}$ which are effectively computable in terms of $q_{1}, q_{2}, \ldots, q_{r}$ and $k$ such that for all $n \in \mathbb{N}, n>k$ we have

$$
[(n-k+1)(n-k+2) \ldots n]_{\mathcal{S}}<c_{8} n^{t-c_{9}}
$$

where $t=\min (k, r)$.

Note that

$$
m \in \mathbb{N}, z>1,\left(m, \prod_{p<z} p\right)=1 \quad \text { imply } \quad \Omega(m) \leq \frac{\log m}{\log z}
$$

since by $\left(m, \prod_{p<z} p\right)=1$ we have

$$
m=\prod_{p^{\alpha} \| m} p^{\alpha} \geq \prod_{p^{\alpha} \| m} z^{\alpha}=z^{\Omega(m)} .
$$

For $n \in \mathbb{N}$, define the non-negative integers $a, b$ and the positive integers $u, v, z$ by

(10.12) $\quad(n-1) n=2^{a} 3^{b} v=u v, \quad 2^{\alpha}\left\|(n-1) n, \quad 3^{b}\right\|(n-1) n, \quad z=2^{a}$, 
so that clearly we have

$$
\Omega(n-1)+\Omega(n)=\Omega(u)+\Omega(v)
$$

and

$$
z \leq n
$$

By (10.11) and (10.12) we have

$$
\Omega(v) \leq \frac{\log v}{\log 5}<\frac{\log \left(n^{2} / u\right)}{\log 5} .
$$

Finally, by using Lemma 4 with $\mathcal{S}=\{2,3\}$, we see that there are effectively computable positive numbers $c_{8}$ and $c_{9}$ such that

$$
u=[(n-1) n]_{\mathcal{S}}<c_{8} n^{2-c_{9}}
$$

so that there are effectively computable positive numbers $c_{10}$ and $n_{4}$ such that

$$
u<n^{2-c_{10}} \quad \text { for } n>n_{4} .
$$

By (10.12) and (10.14), we have

$$
\begin{aligned}
\Omega(u) & =\Omega(z)+\Omega(u / z)=\frac{\log z}{\log 2}+\frac{\log (u / z)}{\log 3} \\
& =\left(\frac{1}{\log 2}-\frac{1}{\log 3}\right) \log z+\frac{\log u}{\log 3} \\
& \leq\left(\frac{1}{\log 2}-\frac{1}{\log 3}\right) \log n+\frac{\log u}{\log 3} .
\end{aligned}
$$

It follows from (10.13), (10.15), (10.16) and (10.17) that for $n>n_{4}$ we have

$$
\begin{aligned}
\Omega(n-1)+\Omega(n) & =\left(\left(\frac{1}{\log 2}-\frac{1}{\log 3}\right) \log n+\frac{\log u}{\log 3}\right)+\frac{\log \left(n^{2} / u\right)}{\log 5} \\
& =\left(\frac{1}{\log 2}+\frac{1}{\log 3}\right) \log n-\left(\frac{1}{\log 3}-\frac{1}{\log 5}\right) \log \left(n^{2} / u\right) \\
& <\left(\frac{1}{\log 2}+\frac{1}{\log 3}-c_{10}\left(\frac{1}{\log 3}-\frac{1}{\log 5}\right)\right) \log n,
\end{aligned}
$$

which proves (10.10).

11. Finally, we will sharpen (9.2) by proving

Theorem 8. For $x \rightarrow \infty$ we have

$$
T(\sigma, x) \leq x\left(M\left(\frac{\sigma(n)}{n}, x\right)+1+O\left((\log \log x)^{-1}\right)\right) .
$$


Note that it is well-known $[7, \S 22.9]$ that

$$
M\left(\frac{\sigma(n)}{n}, x\right)=(1+o(1)) e^{\gamma} \log \log x .
$$

Moreover, note that (11.1) is the best possible apart from the error term, as the following example shows: since

$$
\lim _{x \rightarrow \infty} M\left(\frac{\sigma(n)}{n}, x\right)=\infty,
$$

there are infinitely many integers $x$ such that

$$
\frac{\sigma(n)}{n} \leq \frac{\sigma(x)}{x}=M\left(\frac{\sigma(n)}{n}, x\right) \quad \text { for all } n \leq x .
$$

If $x$ satisfies (11.3), then

$$
\begin{aligned}
T(\sigma, x) & \geq \sigma(x-1)+\sigma(x)=\sum_{d \mid x-1} d+x M\left(\frac{\sigma(n)}{n}, x\right) \\
& \geq 1+(x-1)+x M\left(\frac{\sigma(n)}{n}, x\right)=x\left(M\left(\frac{\sigma(n)}{n}, x\right)+1\right) .
\end{aligned}
$$

To prove Theorem 8 , we need the following lemma:

Lemma 5. For all $\omega>0$ there exist numbers $\varepsilon=\varepsilon(\omega)>0$ and $x_{0}=$ $x_{0}(\omega)$ such that for $x>x_{0}$ we have

$$
\prod_{p \leq \varepsilon \log x}\left(1-\frac{1}{p}\right)^{-1}<M\left(\frac{\sigma(n)}{n}, x\right)-\omega .
$$

Pr o of of Lemma 5. Clearly, it suffices to show that there is a number $R \in \mathbb{N}$ with

$$
R \leq x
$$

and

$$
\frac{\sigma(R)}{R}>\prod_{p \leq \varepsilon \log x}\left(1-\frac{1}{p}\right)^{-1}+\omega .
$$

Indeed, define $R$ in the same way as in the proof of Theorem 4. Then (11.4) holds by (7.4). Moreover, by (2.6) and Mertens' formula we have

$$
\frac{\sigma(R)}{R}=\prod_{p \leq \frac{1}{2} \log x} \frac{\sigma\left(p^{\alpha_{p}}\right)}{p^{\alpha_{p}}}=\prod_{p \leq \frac{1}{2} \log x}\left(1-\frac{1}{p}\right)^{-1}\left(1-p^{-\left(\alpha_{p}+1\right)}\right)
$$




$$
\begin{aligned}
& >\prod_{p \leq \varepsilon \log x}\left(1-\frac{1}{p}\right)^{-1} \prod_{\varepsilon \log x<p \leq \frac{1}{2} \log x}\left(1-\frac{1}{p}\right)^{-1}\left(1-(\log x)^{-1 / 2}\right)^{\pi\left((\log x)^{1 / 2}\right)} \\
& \times \prod_{(\log x)^{1 / 2}<p}\left(1-\frac{1}{p^{2}}\right) \\
& =\prod_{p \leq \varepsilon \log x}\left(1-\frac{1}{p}\right)^{-1} \exp \left((1+o(1))\left(\sum_{\varepsilon \log x<p \leq \frac{1}{2} \log x} \frac{1}{p}-\frac{2}{\log \log x}\right)\right) \\
& =\prod_{p \leq \varepsilon \log x}\left(1-\frac{1}{p}\right)^{-1} \exp \left(\frac{(1+O(1)) \log (1 /(2 \varepsilon))+O(1)}{\log \log x}\right) \\
& =\prod_{p \leq \varepsilon \log x}\left(1-\frac{1}{p}\right)^{-1}+(1+o(1)) e^{\gamma} \log \log x \cdot \frac{\log (1 /(2 \varepsilon))+O(1)}{\log \log x} \\
& =\prod_{p \leq \varepsilon \log x}\left(1-\frac{1}{p}\right)^{-1}+(1+o(1)) e^{\gamma}(\log (1 /(2 \varepsilon))+O(1))
\end{aligned}
$$

which implies (11.5) if $\varepsilon$ is small enough in terms of $\omega$, and this completes the proof of the lemma.

12. Completion of the proof of Theorem 8. We have to show that for all $m \leq x$ we have

$$
\begin{aligned}
& \sigma(m-1)+\sigma(m) \\
& \quad \leq x\left(M\left(\frac{\sigma(n)}{n}, x\right)+1+O\left((\log \log x)^{-1}\right)\right) \quad \text { for all } m \leq x .
\end{aligned}
$$

For all $m \leq x$ we have

$$
\sigma(m-1)+\sigma(m)<m\left(\frac{\sigma(m-1)}{m-1}+\frac{\sigma(m)}{m}\right) \leq x\left(\frac{\sigma(m-1)}{m-1}+\frac{\sigma(m)}{m}\right) .
$$

Thus in order to prove (12.1), it suffices to show that

$$
\frac{\sigma(m-1)}{m-1}+\frac{\sigma(m)}{m} \leq M\left(\frac{\sigma(n)}{n}, x\right)+1+O\left((\log \log x)^{-1}\right) .
$$

If

$$
\max \left(\frac{\sigma(m-1)}{m-1}, \frac{\sigma(m)}{m}\right) \leq \frac{1}{2} \log \log x,
$$

then for $x$ large enough, (12.2) follows from (11.2) and (12.3). Thus we may assume that

$$
\max \left(\frac{\sigma(m-1)}{m-1}, \frac{\sigma(m)}{m}\right)>\frac{1}{2} \log \log x .
$$


Now we will define a finite sequence $\left(u_{0}, v_{0}\right),\left(u_{1}, v_{1}\right), \ldots,\left(u_{z}, v_{z}\right)$ of pairs of positive integers by the following recursion: Define $u_{0}, v_{0}$ so that one of them is equal to $n-1$ and the other is $n$, and

$$
\frac{\sigma\left(v_{0}\right)}{v_{0}} \geq \frac{\sigma\left(u_{0}\right)}{u_{0}} .
$$

Then by (12.4) we have

$$
\frac{\sigma\left(v_{0}\right)}{v_{0}}>\frac{1}{2} \log \log x .
$$

On the other hand, by $(m-1, m)=1$ and (11.2) we have

$$
\begin{aligned}
\frac{\sigma\left(u_{0}\right)}{u_{0}} \cdot \frac{\sigma\left(v_{0}\right)}{v_{0}} & =\frac{\sigma\left(u_{0} v_{0}\right)}{u_{0} v_{0}}=\frac{\sigma((m-1) m)}{(m-1) m} \\
& \leq M\left(\frac{\sigma(n)}{n}, x^{2}\right)=(1+o(1)) e^{\gamma} \log \log x .
\end{aligned}
$$

It follows from (12.5) and (12.6) that for $x$ large enough we have

$$
\frac{\sigma\left(u_{0}\right)}{u_{0}}<6 .
$$

Assume now that $i$ is a non-negative integer and $\left(u_{0}, v_{0}\right), \ldots,\left(u_{i}, v_{i}\right)$ have been defined so that

$$
\begin{array}{cl}
\left(u_{j}, v_{j}\right)=1 & \text { for } j=0,1, \ldots, i, \\
u_{j} v_{j} \mid u_{j-1} v_{j-1} & \text { for } j=1,2, \ldots, i, \\
v_{j}<v_{j-1} & \text { for } j=1,2, \ldots, i, \\
P\left(v_{j}\right)<P\left(v_{j-1}\right) & \text { for } j=1,2, \ldots, i, \\
p\left(u_{j}\right)>p\left(u_{j-1}\right) & \text { for } j=1,2, \ldots, i, \\
\frac{\sigma\left(v_{j}\right)}{v_{j}}>\frac{\sigma\left(v_{j-1}\right)}{v_{j-1}} & \text { for } j=1,2, \ldots, i,
\end{array}
$$

and

$$
\frac{\sigma\left(u_{j}\right)}{u_{j}}+\frac{\sigma\left(v_{j}\right)}{v_{j}} \geq \frac{\sigma\left(u_{j-1}\right)}{u_{j-1}}+\frac{\sigma\left(v_{j-1}\right)}{v_{j-1}} \quad \text { for } j=1,2, \ldots, i
$$

(note that (12.8) holds trivially for $j=0$ ). If $P\left(v_{i}\right)<p\left(u_{i}\right)$, then the construction terminates, i.e., we put $z=i$ so that we have

$$
P\left(v_{z}\right)<p\left(u_{z}\right) .
$$

(Note that (12.11) ensures that the construction terminates in finitely many steps.) If $P\left(v_{i}\right)>p\left(u_{i}\right)$, then write $v_{i}$ and $u_{i}$ as the product of prime powers:

$$
\begin{aligned}
& v_{i}=q_{1}^{\alpha_{1}} \ldots q_{s}^{\alpha_{s}}, \\
& u_{i}=r_{1}^{\beta_{1}} \ldots r_{t}^{\beta_{t}}, \quad q_{1}<\ldots<q_{s}, \\
& r_{1}<\ldots<r_{t}
\end{aligned}
$$


where

$$
P\left(v_{i}\right)=q_{s}>p\left(u_{i}\right)=r_{1} .
$$

(Note that $P\left(v_{i}\right) \neq p\left(u_{i}\right)$ by (12.8).) Then define $u_{i+1}$ and $v_{i+1}$ by

$$
\begin{aligned}
& v_{i+1}=q_{1}^{\alpha_{1}} \ldots q_{s-1}^{\alpha_{s-1}} r_{1}, \\
& u_{i+1}=q_{s}^{\alpha_{s}} r_{2}^{\beta_{2}} \ldots r_{t}^{\beta_{t}} .
\end{aligned}
$$

Then by (12.16), each of (12.8)-(12.12) holds trivially with $i+1$ in place of $j$.

Moreover, if $p$ is a prime number and $\alpha \in \mathbb{N}$, then

$$
\frac{\sigma\left(p^{\alpha}\right)}{p^{\alpha}}=1+\frac{1}{p}+\ldots+\frac{1}{p^{\alpha}}<\left(1-\frac{1}{p}\right)^{-1} \leq\left(1-\frac{1}{2}\right)^{-1}=2
$$

and thus it follows from (12.5) and (12.13) that

$$
\omega\left(v_{i}\right) \rightarrow \infty .
$$

By (12.16) and (12.17) we have

$$
q_{s} \geq r_{1}+2 .
$$

It follows that

$$
\begin{aligned}
\frac{\sigma\left(v_{i+1}\right)}{v_{i+1}} & =\frac{\sigma\left(v_{i}\right)}{v_{i}} \cdot \frac{q_{s}^{\alpha_{s}}}{\sigma\left(q_{s}^{\alpha_{s}}\right)} \cdot \frac{\sigma\left(r_{1}\right)}{r_{1}} \\
& =\frac{\sigma\left(v_{i}\right)}{v_{i}}\left(\sum_{k=0}^{\alpha_{s}} q_{s}^{-k}\right)^{-1}\left(1+\frac{1}{r_{1}}\right) \\
& >\frac{\sigma\left(v_{i}\right)}{v_{i}}\left(1-\frac{1}{q_{s}}\right)\left(1+\frac{1}{r_{1}}\right) \\
& =\frac{\sigma\left(v_{i}\right)}{v_{i}}\left(1+\frac{q_{s}-r_{1}-1}{q_{s} r_{1}}\right)>\frac{\sigma\left(v_{i}\right)}{v_{i}},
\end{aligned}
$$

which proves (12.13) with $i+1$ in place of $j$.

Finally, by (12.18) we have

$$
\begin{aligned}
\frac{\sigma\left(u_{i+1}\right)}{u_{i+1}} & =\frac{\sigma\left(u_{i}\right)}{u_{i}} \cdot \frac{r_{1}^{\beta_{1}}}{\sigma\left(r_{1}^{\beta_{1}}\right)} \cdot \frac{\sigma\left(q_{s}^{\alpha_{s}}\right)}{q_{s}^{\alpha_{s}}} \\
& =\frac{\sigma\left(u_{i}\right)}{u_{i}}\left(\sum_{k=0}^{\beta_{1}} r_{1}^{-k}\right)^{-1}\left(\sum_{k=0}^{\alpha_{s}} q_{s}^{-k}\right) \\
& >\frac{\sigma\left(u_{i}\right)}{u_{i}}\left(1-\frac{1}{r_{1}}\right)\left(1+\frac{1}{q_{s}}\right) \\
& =\frac{\sigma\left(u_{i}\right)}{u_{i}}\left(1-\frac{q_{s}-r_{1}+1}{q_{s} r_{1}}\right)
\end{aligned}
$$




$$
\begin{aligned}
& =\frac{\sigma\left(u_{i}\right)}{u_{i}}\left(1-\frac{\left(q_{s}-r_{1}-1\right)+2}{r_{1} q_{s}}\right) \\
& \geq \frac{\sigma\left(u_{i}\right)}{u_{i}}\left(1-\frac{3\left(q_{s}-r_{1}-1\right)}{r_{1} q_{s}}\right) .
\end{aligned}
$$

Combining (12.19) and (12.20) we obtain

$$
\begin{aligned}
\frac{\sigma\left(u_{i+1}\right)}{u_{i+1}} & +\frac{\sigma\left(v_{i+1}\right)}{v_{i+1}} \\
& >\frac{\sigma\left(u_{i}\right)}{u_{i}}\left(1-\frac{3\left(q_{s}-r_{1}\right)}{q_{s} r_{1}}\right)+\frac{\sigma\left(v_{i}\right)}{v_{i}}\left(1+\frac{q_{s}-r_{1}-1}{q_{s} r_{1}}\right) \\
& =\left(\frac{\sigma\left(u_{i}\right)}{u_{i}}+\frac{\sigma\left(v_{i}\right)}{v_{i}}\right)+\frac{q_{s}-r_{1}-1}{q_{s} r_{1}}\left(\frac{\sigma\left(v_{i}\right)}{v_{i}}-3 \frac{\sigma\left(u_{i}\right)}{u_{i}}\right) .
\end{aligned}
$$

By (7.6), (12.5), (12.7), (12.9) and (12.13) we have

$$
\frac{\sigma\left(v_{i}\right)}{v_{i}} \rightarrow \infty, \quad \frac{\sigma\left(u_{i}\right)}{u_{i}}=O(1),
$$

whence

$$
\frac{\sigma\left(v_{i}\right)}{v_{i}}-3 \frac{\sigma\left(u_{i}\right)}{u_{i}}>0
$$

if $x$ is large enough. (12.14) (with $i+1$ in place of $j$ ) follows from (12.21) and (12.22), and this completes the proof of the existence of a sequence $\left(u_{0}, v_{0}\right),\left(u_{1}, v_{1}\right), \ldots,\left(u_{z}, v_{z}\right)$ with the desired properties.

It follows from (12.5) and (12.13) that

$$
\frac{\sigma\left(v_{z}\right)}{v_{z}}>\frac{\sigma\left(v_{0}\right)}{v_{0}}>\frac{1}{2} \log \log x
$$

where, by (12.10), we have

$$
v_{z}<v_{0} \leq n .
$$

Moreover, by (11.2), (12.8), (12.9) and $u_{0} v_{0}=(m-1) m \leq x^{2}$ we have

$$
\begin{aligned}
\frac{\sigma\left(u_{z}\right)}{u_{z}} \cdot \frac{\sigma\left(v_{z}\right)}{v_{z}} & =\frac{\sigma\left(u_{z} v_{z}\right)}{u_{z} v_{z}} \leq M\left(\frac{\sigma(n)}{n}, x^{2}\right) \\
& =(1+o(1)) e^{\gamma} \log \log x .
\end{aligned}
$$

It follows from (12.23) and (12.25) that

$$
\frac{\sigma\left(u_{z}\right)}{u_{z}}<6 .
$$

If

$$
\frac{\sigma\left(v_{z}\right)}{v_{z}} \leq M\left(\frac{\sigma(n)}{n}, x\right)-5,
$$


then (12.2) follows from (12.14), (12.26) and (12.27). Thus we may assume that

$$
\frac{\sigma\left(v_{z}\right)}{v_{z}}>M\left(\frac{\sigma(n)}{n}, x\right)-5,
$$

whence, by (11.2),

$$
\frac{\sigma\left(v_{z}\right)}{v_{z}} \geq(1+o(1)) e^{\gamma} \log \log x .
$$

Write $v_{z}$ and $u_{z}$ as a product of prime powers:

$$
\begin{aligned}
& v_{z}=q_{1}^{\alpha_{1}} \ldots q_{s}^{\alpha_{s}}, \quad q_{1}<\ldots<q_{s}, \\
& u_{z}=r_{1}^{\beta_{1}} \ldots r_{t}^{\beta_{t}}, \quad r_{1}<\ldots<r_{t} .
\end{aligned}
$$

By (12.15) we have

$$
q_{s}=P\left(v_{z}\right)<p\left(u_{z}\right)=r_{1} .
$$

By Mertens' formula, clearly we have

$$
\begin{aligned}
\frac{\sigma\left(v_{z}\right)}{v_{z}} & =\prod_{i=1}^{s} \frac{\sigma\left(q_{i}^{\alpha_{i}}\right)}{q_{i}^{\alpha_{i}}}=\prod_{i=1}^{s}\left(\sum_{k=0}^{\alpha_{i}} q_{i}^{-k}\right) \\
& <\prod_{i=1}^{s}\left(1-\frac{1}{q_{i}}\right)^{-1} \leq \prod_{i=1}^{s}\left(1-\frac{1}{p_{i}}\right)^{-1}=\prod_{p \leq p_{s}}\left(1-\frac{1}{p}\right)^{-1} .
\end{aligned}
$$

By using Lemma 5 with $\omega=5$, we deduce from (12.28) and (12.31) that there is a positive number $\varepsilon>0$ such that

$$
q_{s} \geq p_{s}>\varepsilon \log x .
$$

It follows from

$$
u_{0} v_{0}=(m-1) m \leq x^{2}
$$

and (12.9) that

$$
u_{z} \leq x^{2} .
$$

This implies that

$$
t=\omega\left(u_{z}\right)<3 \log x
$$

since by the prime number theorem, otherwise we had

$$
\begin{aligned}
u_{z} & =r_{1}^{\beta_{1}} \ldots r_{t}^{\beta_{t}} \geq r_{1} \ldots r_{t} \geq \prod_{i \leq 3 \log x} p_{i} \\
& \geq \prod_{p \leq 3 \log x} p=\exp ((1+o(1)) 3 \log x)>x^{2},
\end{aligned}
$$

which contradicts (12.33). 
It follows from (12.30), (12.32) and (12.34) that

$$
\begin{aligned}
\frac{\sigma\left(u_{z}\right)}{u_{z}} & =\prod_{i=1}^{t} \frac{\sigma\left(r_{i}^{\beta_{i}}\right)}{r_{i}^{\beta_{i}}}=\prod_{i=1}^{t}\left(\sum_{k=0}^{\beta_{i}} r_{i}^{-k}\right) \\
& <\prod_{i=1}^{t}\left(1-\frac{1}{r_{i}}\right)^{-1} \leq \prod_{i=1}^{t}\left(1-\frac{1}{p_{s+i}}\right)^{-1} \\
& \leq \prod_{i<3 \log x}\left(1-\frac{1}{p_{s+i}}\right)^{-1} \\
< & \prod_{\varepsilon \log x<p<4 \log x}\left(1-\frac{1}{p}\right)^{-1} \\
& =\exp \left((1+o(1)) \quad \sum_{\varepsilon \log x<p<4 \log x} \frac{1}{p}\right) \\
& =\exp \left(O\left((\log \log x)^{-1}\right)\right)=1+O\left((\log \log x)^{-1}\right) .
\end{aligned}
$$

It follows from (12.10), (12.14) and (12.35) that

$$
\begin{aligned}
\frac{\sigma(m-1)}{m-1}+\frac{\sigma(m)}{m} & =\frac{\sigma\left(u_{0}\right)}{u_{0}}+\frac{\sigma\left(v_{0}\right)}{v_{0}} \leq \frac{\sigma\left(u_{z}\right)}{u_{z}}+\frac{\sigma\left(v_{z}\right)}{v_{z}} \\
& \leq\left(1+O\left((\log \log x)^{-1}\right)\right)+\max _{n \leq x} \frac{\sigma(n)}{n} \\
& =M\left(\frac{\sigma(n)}{n}, x\right)+1+O\left((\log \log x)^{-1}\right),
\end{aligned}
$$

which proves (12.2), and this completes the proof of the theorem.

Acknowledgements. We would like to thank Professors K. Győry, A. Schinzel, C. L. Stewart and R. Tijdeman for helpful discussions and useful suggestions.

\section{References}

[1] G. J. B abu and P. Erdős, A note on the distribution function of additive arithmetic functions in short intervals, Canad. Math. Bull. 32 (1989), 441-445.

[2] A. S. Bang, Taltheoretiske Undersøgelser, Tidskrift for Math. (5) 4 (1886), 70-80 and $130-137$.

[3] L. E. Dickson, History of the Theory of Numbers, Vol. 1, Chelsea, New York, 1952.

[4] P. Erdős, Remarks on two problems of the Matematikai Lapok, Mat. Lapok 7 (1956), 10-17 (in Hungarian).

[5] -, Remarks on two problems, ibid. 11 (1960), 26-32 (in Hungarian).

[6] P. Erdős et J.-L. Nicolas, Sur la fonction: nombre de facteurs premiers de $N$, Enseign. Math. 27 (1981), 3-21. 
[7] G. H. Hardy and E. M. Wright, An Introduction to the Theory of Numbers, 4th ed., Clarendon Press, Oxford, 1960.

[8] K. Mahler, Lectures on Diophantine Approximations, Part 1: g-adic numbers and Roth's theorem, University of Notre Dame Press, Notre Dame, 1961.

[9] S. Ramanujan, Highly composite numbers, Proc. London Math. Soc. 14 (1915), 347-409.

[10] C. L. Stewart, A note on the product of consecutive integers, in: Colloq. Math. Soc. János Bolyai 34, North-Holland, 1984, 1523-1537.

MATHEMATICAL INSTITUTE

HUNGARIAN ACADEMY OF SCIENCES

REÁLTANODA U. 13-15

H-1053 BUDAPEST, HUNGARY 